\title{
A Graphical Tool for Designing Interactive Video Cognitive Rehabilitation Therapies
}

\author{
J.M. Martínez-Moreno P. Sánchez-González A. García S. González C. Cáceres \\ R. Sánchez-Carrión T. Roig J.M. Tormos and E.J. Gómez
}

\begin{abstract}
Acquired Brain Injury (ABI) has become one of the most common causes of neurological disability in developed countries. Cognitive disorders result in a loss of independence and therefore patients' quality of life. Cognitive rehabilitation aims to promote patients' skills to achieve their highest degree of personal autonomy. New technologies such as interactive video, whereby real situations of daily living are reproduced within a controlled virtual environment, enable the design of personalized therapies with a high level of generalization and a great ecological validity. This paper presents a graphical tool that allows neuropsychologists to design, modify, and configure interactive video therapeutic activities, through the combination of graphic and natural language. The tool has been validated creating several Activities of Daily Living and a preliminary usability evaluation has been performed showing a good clinical acceptance in the definition of complex interactive video therapies for cognitive rehabilitation.
\end{abstract}

Keywords-Interactive Video, Graphical Tool, Activities of Daily Living, Cognitive Rehabilitation, Acquired Brain Injury.

\section{INTRODUCTION}

Brain injury is a life-altering event that affects every area of a person's life including his/ her relationship with family members and close relatives. Acquired Brain Injury (ABI) refers to medical conditions that occur in the brain, altering its function. These conditions include stroke, traumatic brain injury, tumor and other diseases of the brain [1].

Cognitive processes determine individual performance in Activities of Daily Living (ADL) [2]. Thus, cognitive disorders after brain injury result in a loss of autonomy and independence, affecting the patient's quality of life.

Cognitive rehabilitation seeks to increase patients' autonomy and quality of life minimizing or compensating functional disorders showed by ABI patients. Moreover, rehabilitation programs aim to reduce the load, stress and isolation suffered by their family, who become an essential element in such patients' treatment.

Rehabilitation goals must focus on increasing ecological validity [3]. It is strongly recommended that therapeutic activities mimic as much as possible the patient's real environment and needs, merging the rehabilitation into his/her ADL routine. Ultimately, the rehabilitation environment must be adapted, reaching a compromise between realism and flexibility so that therapy can be tailored for any specific impairment profile. Moreover, to ensure maximum efficiency, successful rehabilitation should consider task reproducibility; accurate, effective formative and summative assessment, and the possibility of monitoring the rehabilitation process and the patient's performance [4].

The introduction of disruptive technological solutions in neurorehabilitation leads to higher intensity rehabilitation processes, extending the therapies in an economically sustainable way. The continuous technological development over the last few years on computer processing, graphics/image rendering, display systems, interface and tracking devices, etc.; has increased the application of virtual environment technologies for brain injury rehabilitation. A number of studies have emphasized the benefits of using virtual-based (virtual, augmented and mixed realities, serious games) rehabilitation methods that are both relevant to the patient's real-life context, and that can also be transferred to other ADLs [5][6].

A large sample of works based on serious games development can be found in the literature [7], among which the RehaCom system stands out [8]. Additionally, we can find augmented reality systems, which add virtual elements onto the real environments where the task is performed, such as GenVirtual [9] or ARVe [10]. Finally, Virtual Reality systems can reproduce everyday situations and environments as supermarkets [11], kitchen [12], town neighborhood [13] or even a city [14]. These environments simulate situations of the patients' ADLs encouraging him/her to deal with them as in his/her everyday life.

Interactive Video (IV) can be an appropriate technology to sustain personalized rehabilitation processes based on enhanced environments where real situations of daily living are reproduced [15]. IV refers to any video whose sequences and displayed information depend on the users' 
responses. Interactivity is provided by associating an interaction with any element that appears in the video scenes; the video flow is modified according to the way users interact with them.

IV effectiveness has been proven on the field of learning, where interactive dynamic visualization allows users to adapt the processes to their individual cognitive skills [16][17]. Moreover, some studies have proved that ADL observation treatment is a good rehabilitative approach in stroke patients [18]. The potential of IV lies in their capacity to combine videos portraying real life situations into guided tasks tailored to patient's cognitive profile and needs for achieving therapeutic observation. The combination of both aspects is aimed at reducing to the minimum cognitive requirements needed by the patient to interact with the ADL. Thereby, the neuropsychologist can define every single detail of the environment so that each session focuses only in the specific cognitive functions under rehabilitation. Using IV patient perceives a great quantity of information (cognitive stimulus), either presented as images, texts, audios, actions, etc. Proper structuring and sequencing of these multimedia elements enable the creation of controlled therapeutic situations for the patient to react to and interact with under a therapeutic goal.

Although IV offers many possibilities for the neuropsychologist to set up rehabilitation therapies, as the degrees of freedom increase, so does the complexity and effort required for designing an IV task. Such design complexity along with the inherent technological difficulty of IV management demands powerful tools to assist the neuropsychologist on the creation of IV therapies for cognitive rehabilitation. The main goal of this research work is to present and validate a graphical tool for the design of IV rehabilitation tasks based on ADLs, offering therapists the mean to easily edit the contents and control every aspect of the rehabilitation treatment. By mean of this tool, the therapist has to be able to set the stimuli to be presented, describe each possible interaction of the patient with them, either acceptable or pathological, select new stimuli for each one of them, many of them being "therapeutic" interactions that will be proven and validated, if the patient is able to successfully complete the exercise.

In addition to that, as soon as each interaction is the formal representation of a catalog of therapeutic interventions, each of them absolutely evaluated, it became unavoidable to face the challenge of buildup the system over a taxonomy oriented data base, allowing the emergence of a daily living rehabilitation ontology.

\section{METHODS}

In this research work, a graphical tool has been conceptualized for the therapist to be able to design ADL-based therapies taking clinical advantage of the potential of IV technology. This software allows clinicians to create a therapeutic script of the activity, designing and configuring the stimuli, interactions, assistance and feedback based on the therapeutic goals of the rehabilitation therapy.

The design of the graphical tool has been carried out following the principles of User Centered Design (UCD). The definition of its functional requirements was carried out by an expert panel of 6 neuropsychologists from Institut Guttmann Hospital by means of interviews and questionnaires.

The tool allows the design of the therapeutic script for each ADL, which are structured as a four-level hierarchical model, as shown in Fig. 1. This scheme is based on a therapeutic successive approximation model [3].

A designed ADL is divided into rehabilitation tasks, which in turn are divided into scenes, identifying situations for the generation of therapeutic interventions. Each scene is organized by means of 'pathways', which are composed of 'stimuli', 'actions' and 'responses'. The neuropsychologist can define normal, pathological or rehabilitative pathways.

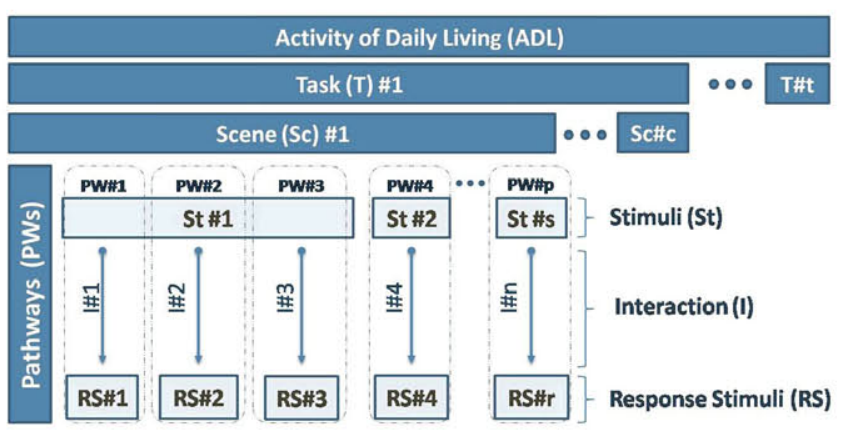

Fig. 1 ADL hierarchical structure

The main functionalities provided by the tool include the creation and edition of the therapeutic ADL script, interactive visualization, and generation of the descriptor file.

Graphical user interface design was performed generating wireframes by means of AxureRP Pro 5.6 software (Fig.2). This example shows the screen where user adds the different script elements. It represents the pathway definition where stimuli are configured and connected defining allowed interaction. 


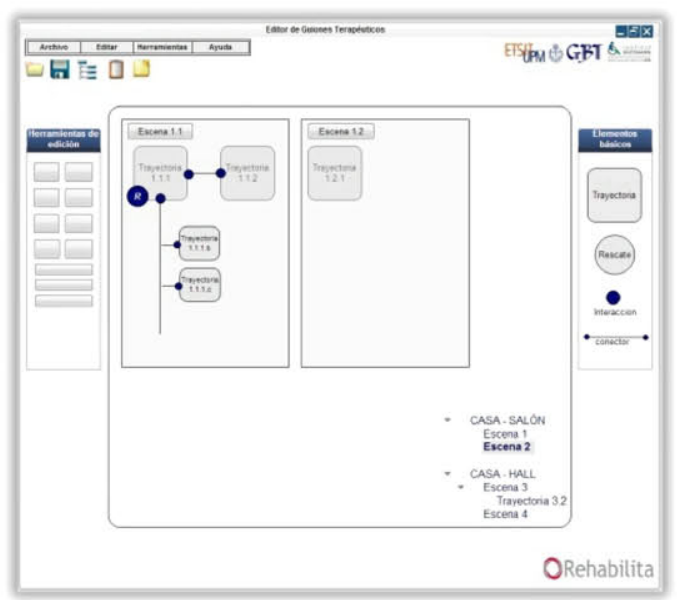

Fig. 2 Wireframe's screen example

The final implementation is based on a Model-ViewController (MVC) framework (Symfony2) and ORM Doctrine in the server side. Extensive use of new functionalities provided by HTML5 combined with Javascript is used for interaction management in the client side.

A preliminary usability evaluation by likert-type questionnaires has been performed on the complete graphical tool in which five specialists from Guttmann hospital were involved. In order to validate the tool, an ADL 'buying bread' activity using IV technology was developed [15].

\section{RESULTS}

Figure 3 shows an example of the graphical user interface (GUI) in which the creation and visualization of an ADL exercise's task is displayed. Three main areas are shown on this screen: title task bar, menus bar and tools bar (top); structure task, it is a list of the task elements (right); and (center) the designing board, where boxes represent task's scenes and lines represent pathways.

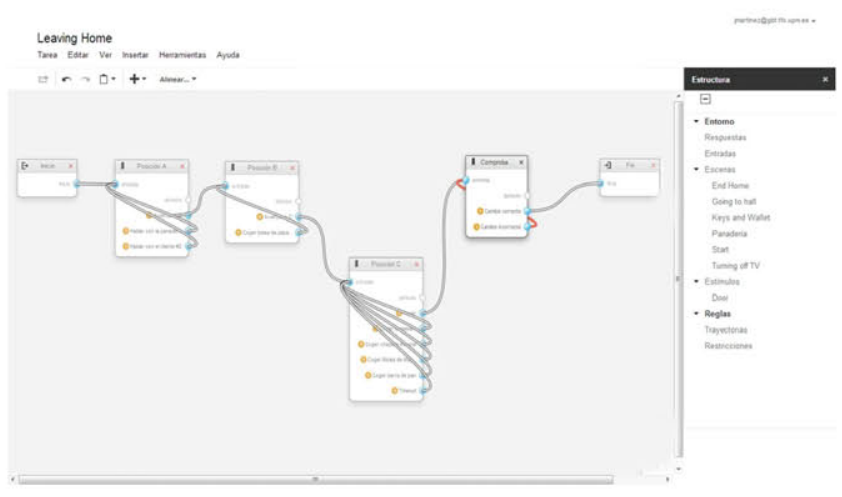

Fig. 3 Main Screen
The ADL 'buying bread' script was designed using the tool by Guttmann Hospital neuropsychologists and recorded with amateur actors in outdoor and indoor real settings (see Fig. 4). The ADL virtual environment allows patients to navigate through a series of scenes representing the different steps to follow in order to reach a final goal, from sitting on the couch at home (initial state) to buying bread at the bakery (end state) [15]. Throughout the task, patients are required to make decisions: choosing the next step, answering questions or interacting with other characters. Every single action in the task is under therapist control, who has previously adjusted the video scenes sequence to the cognitive capabilities required by the patient. Thus, all stimuli in the scenes may be preprogrammed by therapists in order to help the patient or even distract him/her from completing a task.

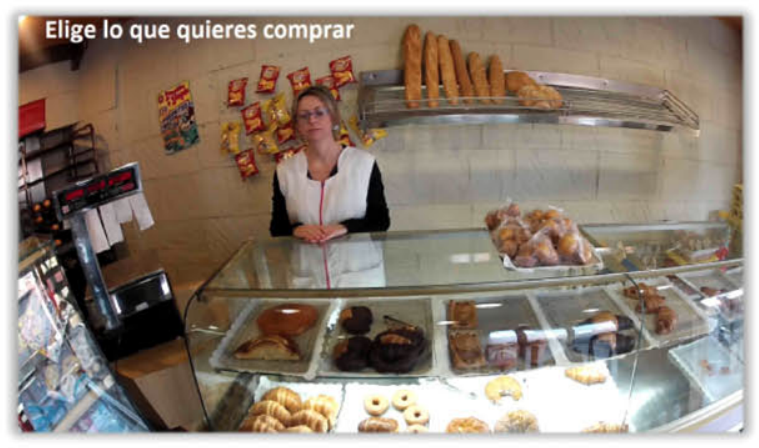

Fig. 4 Bakery task in ADL 'Buying Bread'.

Global results for the validation of the therapeutic designing graphical tool are shown in Fig 5. Ease of learning, ease of use, satisfaction, efficiency and efficacy have been analyzed, in many cases showing good preliminary results.

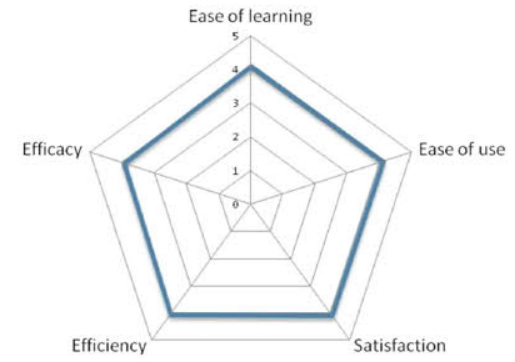

Fig. 5 Evaluation results. (1: Slightly satisfied; 5: Extremely satisfied)

\section{CONCLUSIONS}

Cognitive neurorehabilitation therapies help brain injury patients enhance their ability to move through daily life by recovering or compensating for damaged cognitive func- 
tions. A restorative approach helps patients reestablish cognitive function, while compensatory approaches help the individual adapt to an ongoing impairment.

This paper has presented the design and development of a graphical tool to create cognitive rehabilitation ADL therapies base on interactive video technology. IV provides several advantages compared to traditional virtual environment technologies (virtual reality).Virtual environment technologies are offering many new possibilities to generate cognitive rehabilitation therapy interventions adapted to the patients' status and needed treatment.

Using real image provides more similarity between rehabilitation environment and patient's daily life. Organizing the video by pathways, neuropsychologist is allowed to adapt ADL to any cognitive profile. Thus, required compromise between realism and flexibility is reached. IV allows control of interactivity and screen movements, and reduces the risk of sickness symptoms (i.e. cybersickness).

The developed tool can be a valuable asset for the creation of cognitive rehabilitation therapies based on IV activities of daily living. It provides a powerful software to help neuropsychologists to conceptualize the therapeutic goals for brain injury patients and to create interactive videos supporting the rehabilitation processes.

Preliminary usability evaluation results of the therapies designing tool have shown that it is intuitive, ease to use and accessible to any clinician regardless of their experience with software developing tools. Further studies are required to confirm the clinical usage of the tool and the potentiality of interactive video environments for cognitive rehabilitation of brain injury patients.

\section{ACKNOWLEDGMENT}

This research has been partially funded by the project CENIT-E 'REHABILITA' CEN-20091043.

\section{REFERENCES}

1. Woodward B. Understanding Brain Injury. A guide for the Family. 2008. MFMER.

2. Marcotte T, Cobb J, Kamat R. Neuropsychology and the Prediction of Everyday Functioning. In Neuropsychology of Everyday Functioning. (2010). pp. 5-39.

3. Wilson B, Herbert C, Shiel A. Behavioural Approaches in Neuropsychological Rehabilitation: Optimising Rehabilitation Procedures. (2003). ISBN 1-84169-183-6.

4. Lee JH, Ku J, Cho $\mathrm{W}$ et al. A virtual reality system for the assessment and rehabilitation of the activities of daily living. Cyberpsychol Behav.(2003) Aug;6(4). Pp 383-8.
5. Laver K, George S, Thomas S, Deutsch JE, Crotty M. Cochrane review: virtual reality for stroke rehabilitation. Eur J Phys Rehabil Med. 2012 Sep;48(3):523-30. Epub 2012 Jun 20.

6. Moreira MC, de Amorim Lima AM, Ferraz KM, Benedetti Rodrigues MA. Use of virtual reality in gait recovery among post stroke patients: a systematic literature review. DisabilRehabil Assist Technol. 2013 Apr 24.

7. Rego P, Moreira P.M., Reis L.P. Serious games for rehabilitation: A survey and a classification towards a taxonomy. Proceeding of the 5th Iberian Conference on Information Systems and Technologies (CISTI), 2010. ISBN 978-1-4244-7227-7.

8. Fernández E et al. Clinical impact of RehaCom Software for cognitive rehabilitation of patients with acquired brain injury. MEDICC rev. [online]. 2012, vol.14, n.4, pp. 32-35. ISSN 1555-7960.

9. Correa A, Grasielle D, et al. GenVirtual: An Augmented Reality Musical Game for Cognitive and Motor Rehabilitation. IEEE, 2007. Virtual Rehabilitation. pp. 1-6. ISBN: 978-1-4244-1204-4

10. Richard E, et al. Augmented Reality for Rehabilitation of Cognitive Disabled Children: A Preliminary Study. IEEE, 2007. Virtual Rehabilitation, 2007. pp. 102-108.

11. Rand D, Weiss PL, Katz N. Training Multitasking in a Virtual Supermarket: A Novel Intervention After Stroke. Occupational Therapy September/October 2009 vol. 63 no. 5 535-542.

12. Cao X, Douguet AS, Fuchs $P$, Klinger. Designing an ecological virtual task in the context of executive functions: a preliminary study. Proc. 8th Intl Conf. Disability, Virtual Reality \& Associated Technologies. (2010). ISBN 9780704915022

13. E. Klinger E, Kadri A, Sorita E, Le Guiet JL et al. AGATHE: A tool for personalized rehabilitation of cognitive functions based on simulated activities of daily living. IRBM (2013) pp 113-118.

14. Dores AR, Carvalho IP, Mendes L. Virtual City: Neurocognitive rehabilitation of Acquired Brain Injury. Proceeding of the $7^{\text {th }}$ Iberian Conference on Informtation Systems and Technologies (CISTI) (2012). ISBN: 978-1-4673-2843-2.

15. Martínez-Moreno JM, Solana J, Sánchez R, et al.Monitoring visual attention on a neurorehabilitation environment based on Interactive Video. Proceedings of the International Conference on Recent Advances in Neurorehabilitation (2013). pp 182-185

16. Schwan S,Riempp R. The cognitive benefits of interactive videos: learning to tie nautical knots. Learning and Instruction 14 (2004), 293-305.

17. Martin M, Weigand S, Heier A, Schwan S. Learning with videos vs. learning with print: The role of interactive features. Learning and Instruction 21 (2011), 687-704.

18. Franceschini M, Agosti M, Cantallo A, et al, Mirror neurons: actions observation treatment as a tool in stroke rehabilitation. European Journal of physical and rehabilitation medicine 46 (2010), 517-523.

\author{
Author: José María Martínez Moreno \\ Institute: Biomedical Engineering and Telemedicine Centre \\ Street: Av. Complutense 30 \\ City: Madrid \\ Country: Spain \\ Email: jmartinez@gbt.tfo.upm.es
}

